\title{
High Energy Particles at and from the Sun
}

\author{
Nicole VILMER \\ Observatoire de Paris, Section de Meudon, DASOP, URA 324, CNRS
}

(Received August 31, 1990)

\begin{abstract}
A large amount of the energy released during solar flares is converted into high energy particle acceleration: electrons and ions. These particles are detected either in the interplanetary space or through the radiations that they produce in the solar atmosphere (radio, X-ray and $\gamma$-ray emissions). After a brief discussion of the hard Xray and $\gamma$-ray emission processes, we focus on the observational constraints on the accelerated particles: occurrence of ion acceleration in solar flares, time scale for particle acceleration, transport from the acceleration site to the emitting regions. The relative abundances of the accelerated nuclei and in the interaction site deduced from $\gamma$-ray spectroscopy are discussed. Finally the relationship between gamma-ray line and solar energetic events will be examined.
\end{abstract}

\section{Introduction}

A large number of particles are accelerated during solar flares (typically $10^{36}$ or more electrons with energies above $30 \mathrm{keV} ; 10^{33}-10^{34}$ protons above $30 \mathrm{MeV}$ ). Some of these particles interact in the solar atmosphere producing radio emission in the corona and X-ray $\gamma$-ray emissions in the denser regions. While X-rays and $\gamma$-rays are the most direct diagnostics of the accelerated particles, radio observations provide constraints on the magnetic topology in the corona. Some of the accelerated particles may also escape into the interplanetary space where they are directly detected (solar energetic particle events or SEP). Two kinds of informations can be deduced from these two sets of observations: characteristics of the accelerated particles themselves and of the ambient medium. The main difficulty to deduce the characteristics of the acceleration processes is thus to evalute in the observations the effects which are due to the transport of the accelerated particles from the acceleration region in the low corona to the usually dense chromospheric X-ray and $\gamma$-ray interaction sites. In the case of SEP events, the observations must be deconvolved from the effects of coronal and interplanetary propagation.

\section{X-Ray and $\gamma$-Ray Emitting Particles}

Figure 1 shows the best X-ray and $\gamma$-ray spectrum observed from $\sim 400 \mathrm{keV}$ to $10 \mathrm{MeV}$ during the intense, long duration ( $\sim 30$ minutes) gamma ray line (GRL) flare of April 27, 1981. It consists of several nuclear deexcitation lines from $\mathrm{Fe}, \mathrm{Mg}, \mathrm{Ne}, \mathrm{Si}, \mathrm{C}, \mathrm{O}$ and $\mathrm{N}$, of the delayed lines from neutron radiative capture at $2.2 \mathrm{MeV}$ and from electron-positron annihilation at $511 \mathrm{keV}$. These lines are superposed on a continuum made of the same kinematically broadened deexcitation lines and of a bremsstrahlung component extending 


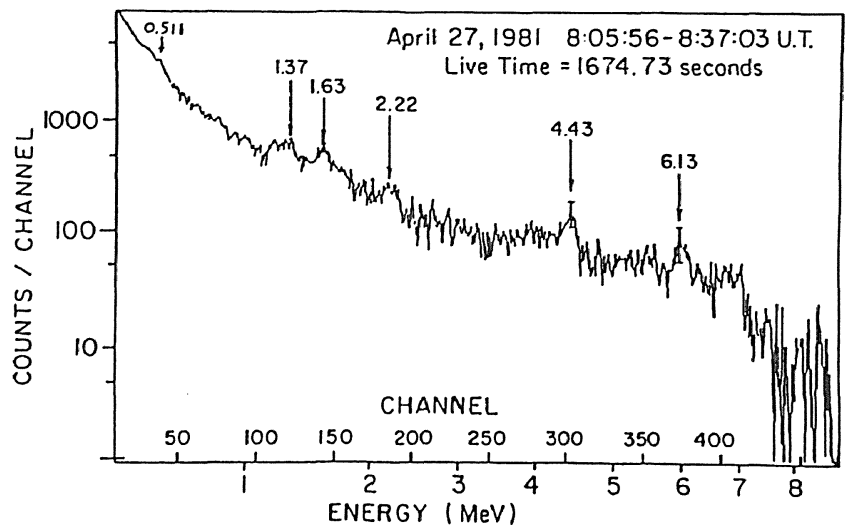

Fig. 1. The $\gamma$-ray spectrum observed by the SMM-GRS for the April 27, 1981 flare. $\gamma$-ray lines are respectively: $6.13 \mathrm{MeV}\left({ }^{16} \mathrm{O}\right), 4.43 \mathrm{MeV}\left({ }^{12} \mathrm{C}\right), 2.22 \mathrm{MeV}(\mathrm{n}), 1.64 \mathrm{MeV}\left({ }^{20} \mathrm{Ne}\right), 1.37 \mathrm{MeV}\left({ }^{24} \mathrm{Mg}\right)$, $0.511 \mathrm{MeV}\left(\mathrm{e}^{+}\right)$(after Chupp, 1982).

above $1 \mathrm{MeV}$. For other events, this last component may extend to energies greater than 10 $\mathrm{MeV}(\sim 10 \%$ of the flares observed from 1980 to 1989$)$.

The $\gamma$-ray lines are unambiguously produced by nuclear reactions of energetic ions $(>10$ $\mathrm{MeV} /$ nucleon) with the ambient medium. These reactions result in excited nuclei with short life times $\left(\sim 10^{-11} \mathrm{sec}\right)$ producing the deexcitation "prompt" $\gamma$-ray lines and in secondary products such as positrons, pions and neutrons giving rise respectively to the delayed 511 $\mathrm{keV}$ annihilation line, a broad emission around $70 \mathrm{MeV}$ and the delayed $2.2 \mathrm{MeV}$ line (e.g. RAMATY and MURPHY, 1987). For higher energetic primary ions ( $>100 \mathrm{MeV} / \mathrm{nucl})$, the energetic neutrons produced can also be directly detected in the interplanetary space $(>50 \mathrm{MeV}$ neutrons) or produce neutron decay protons ( $>20 \mathrm{MeV}$ neutrons) (CHUPP, 1988).

There are still some controversies on the nature of the primary particles emitting the bremsstrahlung component above a few dekakeV. It is generally attributed to energetic electrons with energies greater than $30 \mathrm{keV}$. However, although a good agreement is generally obtained between the numbers of microwave and X-ray emitting electrons, problems arise from the large number of electrons needed to produce observed X-ray fluxes (beam return currents, acceleration volume,...) (e.g. VILMER, 1987). This leads to an alternative interpretation where X-rays are produced by more efficient energetic protons $(>60$ $\mathrm{MeV}$ ) (HERISTCHI, 1986). The number of energetic particles is thus reduced by a factor $\sim 2000$. However, there is still unresolved discrepancy between the number of protons deduced from the continuum or from the $\gamma$-ray lines. It is not yet clear whether this is due to a break in the proton spectrum between the low energy producing part $(>10 \mathrm{MeV})$ and the higher energy part of the spectrum $(>60 \mathrm{MeV})$ responsible for the X-ray continuum. In the following, we shall consider that the $\mathrm{X}$-ray continuum is produced by energetic electrons.

\subsection{Occurrence of ion acceleration in solar flares}

During the last activity cycle, SMM observations have shown that ion acceleration at energies between 10-100 MeV/nucleons is relatively common during flares. This is evidenced by the observed rough correlation between the electron content (estimated from the 
bremsstrahlung total flux above $270 \mathrm{keV}$ ) and the nuclear content (estimated from the 4-8 $\mathrm{MeV}$ excess flux above bremsstrahlung) (CHUPP, 1984). However, some uncertainties in the nuclear content could arise from the method used to separate the different components in this energy band (MURPHY et al., 1990). An important question is also to check whether such a correlation still holds for weaker events which could be observed with more sensitive instruments. Moreover, the observation in the new cycle of a few intense events with very weak nuclear line emission and a strong continuum up to $60 \mathrm{MeV}$ (RIEGER et al., 1990 in CHUPP, 1990) has raised new questions about the relative amount of electrons and ions accelerated during flares. Does this ratio depend on the acceleration mechanism or on the physical characteristics of the acceleration site which could vary from one flare to the other? New observations of such events are necessary to answer this question.

\subsection{Time scale for particle acceleration}

The fastest time scales observed in the radio and X-ray domains, characteristic of the acceleration/interaction time scales of deka-keV electrons are of the order of a few ten milliseconds (DESAI et al., 1987; GÜDEL and BENZ, 1990). The temporal resolution of the $\gamma$-ray detectors is smaller. However, a simultaneous production of energetic electrons ( $>$ a few 100 $\mathrm{keV}$ ) and ions (10-100 MeV/nucleons), as detected by the 4-6 MeV flux assumed to be mainly produced by nuclear radiation, is generally observed on a time scale of 1 second (see e.g. KANE et al., 1986). Moreover, as shown by direct neutron detection in space, nucleons can be accelerated up to a few $\mathrm{GeV} /$ nucleons on time scales of a few tens of seconds at the beginning of a flare (CHUPP, 1982). The flare acceleration mechanism must be thus efficient and rapid. Although in most events, 4-6 MeV and X-ray fluxes reach their maxima simultaneously (within the detector time resolution), in some events $(\sim 8)$ there are delays of a few seconds to a few tens of seconds between the 4-6 MeV and the low energy X-ray maxima. These delays have a tendency to increase with flare duration (YOSHIMORI, 1989). Two interpretations have been proposed either in terms of a delayed (by a few seconds) second step acceleration of ions and high energy electrons as compared to low energy ones or in terms of the result of particle transport between the acceleration and interaction sites. The sensitivity of the available detectors has not allowed yet to study the temporal evolution of the individual $\gamma$-ray lines but only of the $4-6 \mathrm{MeV}$ band so that all these results should be further confirmed with more sensitive experiments.

\section{$2.3 \gamma$-ray emission models including transport}

Different $\gamma$-ray models have been developed to evaluate the effects on the temporal and spectral evolutions of $\gamma$-ray emissions of particle transport. Energetic ions are usually injected (generally isotropically) in an ionized coronal magnetic loop with a uniform density and magnetic field. Some of these ions precipitate in the loss cone created by the rapidly converging magnetic field at the transition between the corona and the neutral chromosphere where the ambient density suddenly increases. These ions reach the dense layers of the atmosphere where they produce the different $\gamma$-ray emissions. The loss cone is usually fed by ion scattering due to wave-particle interactions in the ionized coronal trap. Energy losses of the energetic particles in the corona and during their propagation towards the dense layers as well as the effects of partial trapping are taken into account to relate the energetic protons in the interaction site to the injection spectrum. The models differ by the methods used to study this relationship (either a Monte Carlo simulation of the Fokker-Planck equation or analytical solutions of a continuity equation) and by the exact assumptions on the loop 
geometry. However, they generally treat particle acceleration as a black box, independently from the particle transport. Future progress must thus be done in the simultaneous treatment of acceleration and transport in the same magnetic structure. Different predictions have been made with these models: decay phase of $\gamma$-ray lines (RAMATY et al., 1990a) and temporal evolutions of neutron production and $\pi^{\circ}$ decay radiation in the case of an impulsive ion injection (KoCHAROV et al., 1990); relative X-ray and $\gamma$-ray timing in the case of a longduration injection (HuLOT et al., 1989). In the last case, X-ray producing electrons and $\gamma$-ray producing ions are injected simultaneously in the same structure in order to study, independently from the acceleration timing, the effects of particle transport on the temporal evolutions of the radiations.

This model has been applied to one main peak of the April 27, 1981 event where a delay of $\sim 40$ seconds is observed between the maxima of the $4.1-6.4 \mathrm{MeV}$ and $110-136 \mathrm{keV}$ energy bands (Fig. 2(a)). The temporal evolutions of the X-ray fluxes at different energies and that of the delayed 4.1-6.4 MeV considered as a good indication of the timing of the prompt $\gamma$-ray lines can be reproduced without a second step acceleration of energetic ions if electrons and ions are injected simultaneously and continuously in the same magnetic loop of mean density $n=510^{10} \mathrm{~cm}^{-3}$ (Figs. 2(b)-2(c)). Particle transport acting differently on electrons and ions are responsible for the different temporal evolutions (see HULOT et al., 1991 for more details). This does not rule out completely the existence of a delayed ion acceleration but shows that the effects of particle transport must necessarily be taken into account for any detailed analysis of X-ray and $\gamma$-ray time profiles. For that peculiar event, $\mathrm{X}$-ray emission comes predominantly from the trapped electrons while $\gamma$-ray lines are produced either essentially by trapped protons (curve 1 of Fig. 2(c)) or by both trapped and precipitated protons (curve 2 of Fig. 2(c)). The available data do not allow to decide between both interpretations. However, an interesting consequence is that, even in case $2,30 \%$ of the $\gamma$-ray line flux is produced in the corona.

\section{$2.4 \gamma$-ray line spectroscopy}

Relative abundances of some elements in the interaction site (O, C, Mg, $\mathrm{Si}, \mathrm{Fe}, \mathrm{Ne}, \ldots)$ as well as relative abundances of the same accelerated nuclei can be deduced from the comparison of observed $\gamma$-ray spectra with predictions made on the contributions of the narrow $\gamma$-ray lines produced by light energetic ions interacting with heavy ambient nuclei and of the same kinematically broadened lines from the interaction of heavy energetic nuclei on the light ambient elements. Such a study has been done for the April 27, 1981 event (RAMATY et al., 1990b). The deduced abundances in the interaction site differ from photospheric ones in that $\mathrm{Mg} / \mathrm{C}, \mathrm{Si} / \mathrm{C}, \mathrm{Fe} / \mathrm{C}$ ratios are greater than photospheric values and closer to coronal values. The $\mathrm{O} / \mathrm{C}$ ratio is consistent with both photospheric and coronal values. Moreover the $\mathrm{Ne} / \mathrm{C}$ ratio, much greater than the photospheric value, is also inconsistent (greater) with the coronal one. The question that arises from these results concerns the location of the prompt $\gamma$-ray interaction site:

- is it, as generally assumed, in the chromosphere? Chromospheric abundances, apart from Neon, are then closer to coronal ones than to photospheric ones.

- is it, for that peculiar event, partly in the corona, as suggested by the results of the study presented in $c$ ? This interpretation naturally explains most of the properties of the deduced abundances. 


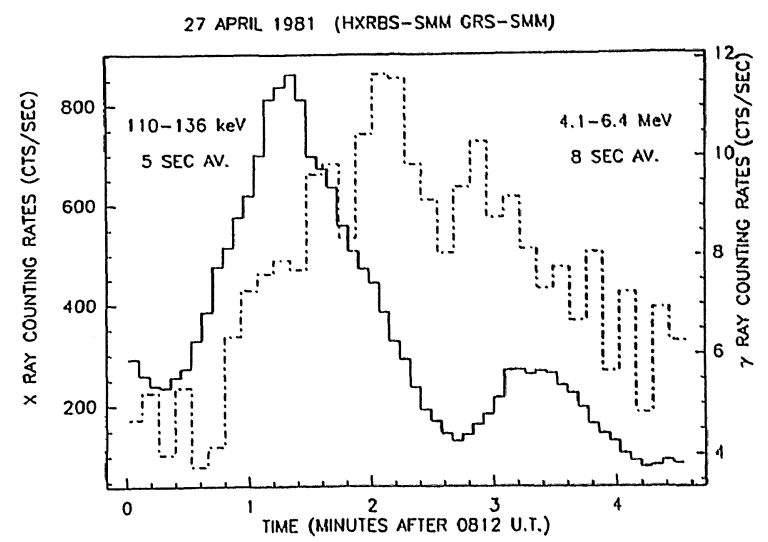

Fig. 2(a). Time evolutions of 110-136 keV (from HXRBS-SMM) and 4.1-6.4 MeV (from GRS-SMM) counting rates for the peak at $\sim 0813$ U.T. of the April 27, 1981 event.
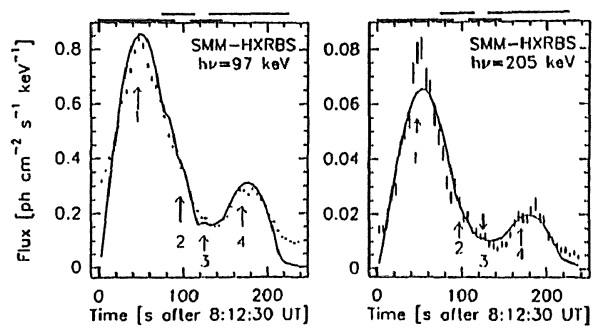

Fig. 2(b). Comparison between observed (error bars) and expected (full lines) hard X-ray times profiles at $97 \mathrm{keV}, 205 \mathrm{keV}$.

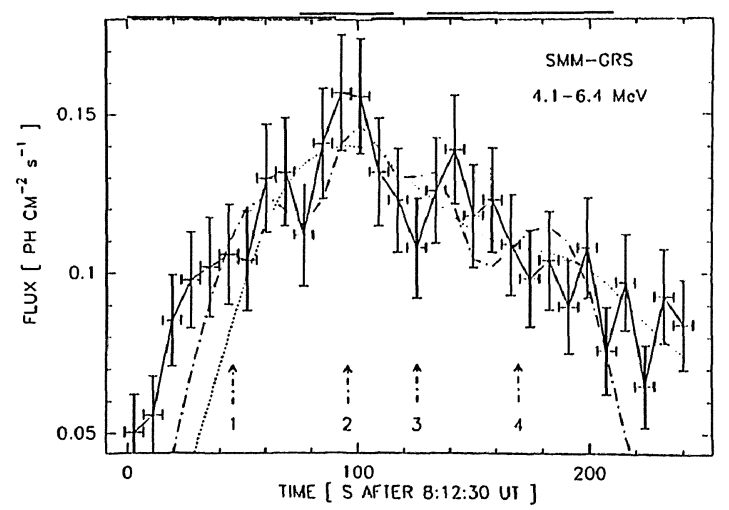

Fig. 2(c). Comparison between observed (-) and expected 4.1-6.4 MeV time profiles in the case of a perfect ion trap (…) and of a leaky trap (.--) with $\alpha_{0}{ }^{2} / L=10^{-6} \mathrm{~km}^{-1}\left(\alpha_{0}\right.$ is the loss cone angle and $L$ is the characteristic length of the coronal loop) (after HuLot et al., 1991). 
However, the enrichment of some of the elements compared to photospheric values must be understood wherever the interaction site lies (chromosphere or corona or both). It may be interpreted as the result of a magnetic filter acting between the photosphere and the corona, at a level where the photospheric magnetic field diverges and coronal structures begin to form (VAUCLAIR and MEYER, 1985). At that level, elements with a low first ionization potential such as $\mathrm{Mg}, \mathrm{Si}, \mathrm{Fe}$ are partly ionized and thus magnetically frozen, which prevents them from being mixed again in the supergranular flow pattern. This results in an enhancement of these elements in the chromosphere or corona compared to photospheric values. However, this does not explain the high $\mathrm{Ne} / \mathrm{C}$ ratio found from $\gamma$-ray spectroscopy. Such a value has been deduced so far only on one event and other estimates are necessary to further conclude. Moreover, observations obtained with a better spectral resolution (as with Ge detectors) would allow to identify more clearly the different $\gamma$-ray lines and thus give stronger constraints on the deduced abundances.

The relative abundances of the accelerated nuclei deduced for that event are found to be inconsistent with the abundances measured for large proton flares in the interplanetary space, but consistent with the ones of ${ }^{3} \mathrm{He}$ rich events in space (RAMATY et al., 1990b). This result is further discussed below.

\section{3. $\gamma$-Ray Line (GRL) Events and Solar Energetic Particle (SEP) Events}

The link between GRL flares and interplanetary proton events has been extensively studied by CLIVER et al. (1989). The principal result is a lack of clear correlation between the 4-8 MeV GRL fluence characteristic of the number of $\sim 10 \mathrm{MeV}$ protons trapped at the sun and the peak fluxes of $\sim 10 \mathrm{MeV}$ protons in the interplanetary space, even for well connected events. This is primarily due to large proton events associated with flares with no detectable GRL emission. The ratio of $\sim 10 \mathrm{MeV}$ protons producing GRL emission to the number of $\sim 10 \mathrm{MeV}$ protons detected in space can thus vary by several orders of magnitude from one flare to the other. However, there may be some uncertainties in the proton numbers deduced either from GRL production models or from the modelling of proton transport in the interplanetary space.

There is a clear tendency for GRL events to be associated with "impulsive" flares (duration less than 10 minutes as defined by the e-folding time of the associated soft X-ray emission) while interplanetary proton events are rather associated with "extended" flares (duration greater than 10 minutes). Furthermore, the existence of two classes of interplanetary particle events has been reported (see e.g. CANE et al., 1986; RAMATY et al., 1990b; MOSES et al., 1989):

- SEP events associated with "impulsive" soft X-ray events (i.e. more probably associated with GRL flares) have a tendency to have a higher e/p ratio in space (5 GRL flares among 7 correspond to high $\mathrm{e} / \mathrm{p}$ ratios), to be enriched in ${ }^{3} \mathrm{He}$ and to have a higher $\alpha / \mathrm{p}$ ratio. The abundances of heavy elements are enhanced compared to $\mathrm{C}$ and $\mathrm{O}$ nuclei as in the case of the abundances of the accelerated nuclei deduced for one event from $\gamma$-ray spectroscopy. For 2 GRL events, the SEP events tend to have a hard particle spectrum. This class of event is characterized by a relatively weak particle flux in space and corresponds to electron events in space exhibiting a spectral hardening of the $0.1-100 \mathrm{MeV}$ electron spectra at high energies.

- The second class of SEP events associated with "extended" soft X-ray events (Long Duration Events) gives large interplanetary proton fluxes and electron spectra with no break. As shown before, there is a weak connection with GRL flares. 
Thus, GRL and large proton events seem to be loosely related. The interpretation of this lack of association is however not so clear: is there only one acceleration mechanism acting on the Sun with escape conditions from the acceleration site to the interplanetary space varying from flare to flare or do these two classes of events correspond to two different acceleration mechanisms, the first one efficient in closed magnetic structures, thus responsible for large GRL events, while the second one operates in open magnetic structures? A shock acceleration operating high in the corona has been often suggested for this last case.

\section{Conclusion}

New ideas on the acceleration of high energy particles during solar flares have emerged from the large amount of observations at high energies obtained during the last ten years. However, some unknowns remain as the relative amount of electrons and ions accelerated during flares. This is one of the problems to be solved during this maximum with new observations. Some theoretical improvements are also necessary to predict these relative numbers for the different acceleration mechanisms. The relative timing of ion and electron acceleration should be also further studied with more sensitive experiments allowing to follow the temporal evolutions of the $\gamma$-ray lines themselves. Moreover, the effects of particle transport from the acceleration to the interaction sites should be studied systematically. Models should be improved in treating coherently particle acceleration and transport which could occur simultaneously in the same magnetic structures. Finally, new constraints could be obtained on the angular distribution of the emitting particles from $\gamma$-ray observations obtained with high spectral resolution. These observations will also provide further constraints on the abundances in the $\gamma$-ray emitting region and of the accelerated nuclei.

GRL and large proton events seem to be loosely related and to correspond to 2 SEP classes. However, it is not clear how strong are the correlations between the properties characterizing the first class, since these relations hold on few events. Furthermore, there is a lack of events studied in a coherent way such as a close examination of the different emissions at the sun and of the different components of the particle event. The link between the deep layers of the atmosphere and the particle injection site in space should be examined in details with the analysis of e.g., radio observations which give constraints on the magnetic topology at different heights. This would give new clues to the differences between gamma ray line and interplanetary proton events: is the magnetic topology or size of the region where the acceleration takes place different or are there two different acceleration mechanisms?

\section{REFERENCES}

CAne, H. V., R. E. Mc Guire, and T. T. von Rosenvinge, Astrophys. J., 301, 448, 1986.

Chupp, E. L., in Gamma-Ray Transients and Related Phenomena, edited by R. E. Lingenfelter, H. S. Hudson, and D. M. Worall, A.I.P., New York, 77, 363, 1982.

Chupp, E. L., Ann. Rev. Astron. Astrophys., 22, 359, 1984.

Chupp, E. L., Solar Phys., 118, 137, 1988.

Chupp, E. L., Science, 250, 229, 1990.

Cliver, E. W., D. J. Forrest, H. V. Cane, D. V. Reames, R. E. Mc Guire, T. T. von Rosenvinge, S. R. KANE, and R. J. MAC Dowall, Astrophys. J., 343, 953, 1989.

Desai, V., C. Kouveliotou, C. Barat, K. Hurley, M. Niel, R. Talon, G. Vedrenne, I. V. Estulin, and V. C. Dolidze, Astrophys. J., 319, 567, 1987. 
GÜDEL, M. and A. O. BENZ, Astron. Astrophys., 231, 202, 1990.

Heristchi, D. J., Astrophys. J., 311, 474, 1986.

Hulot, E., N. Vilmer, and G. Trottet, Astron. Astrophys., 213, 383, 1989.

Hulot, E., N. Vilmer, E. L. Chupp, B. R. Dennis, and S. R. Kane, Astron. Astrophys., 1991 (to be published).

Kane, S. R., E. L. Chupp, D. J. Forrest, G. H. Share, and E. Rieger, Astrophys. J. Letters, 300, L95, 1986.

Kocharov, L. G. and G. A. Kovaltsov, Solar Phys., 125, 67, 1990.

Moses, D., W. Dröge, P. Meyer, and P. Evenson, Astrophys. J., 346, 523, 1989.

Murphy, R. J., G. H. Share, J. R. Letaw, and D. J. Forrest, Astrophys. J., 358, 298, 1990.

Ramaty, R., J. A. Miller, X. M. HuA, and R. E. Lingenfelter, Astrophys. J. Supplement, 73, 199, 1990.

Ramaty, R. and R. J. Murphy, Space Science Reviews, 45, 213, 1987.

Ramaty, R., R. J. MurPhy, and J. A. Miller, in Particle Astrophysics, edited by V. Jones, F. Kerr, and J. Ormes, p. 143, A.I.P., New York, 1990.

Vauclair, S. and J. P. MeYer, 19th Intern. Cosmic Ray Conf., 4, 233, 1985.

Vilmer, N., Solar Phys., 111, 207, 1987.

Yoshimori, M., Space Sci. Rev., 51, 85, 1989. 